OPEN ACCESS

Edited by:

Wei Wei,

Anhui Medical University, China

Reviewed by:

Youcai Deng,

Third Military Medical University,

China

Huichang Bi,

Sun Yat-sen University, China

Yan Huang,

Anhui Medical University, China

*Correspondence:

Hong Wu

hongw@aliyun.com,

wuhongprof@aliyun.com

†These authors have contributed equally to this work and are co-first

authors.

Specialty section: This article was submitted to Inflammation Pharmacology, a section of the journal

Frontiers in Pharmacology

Received: 31 October 2017

Accepted: 30 January 2018

Published: 15 February 2018

Citation:

Deng R, Li F, Wu H, Wang W-y, Dai L, Zhang Z-r and Fu J (2018)

Anti-inflammatory Mechanism of Geniposide: Inhibiting the Hyperpermeability of Fibroblast-Like Synoviocytes via the RhoA/p38MAPK/NF- $\kappa$ B/F-Actin

Signal Pathway.

Front. Pharmacol. 9:105.

doi: 10.3389/fphar.2018.00105

\section{Anti-inflammatory Mechanism of Geniposide: Inhibiting the Hyperpermeability of Fibroblast-Like Synoviocytes via the RhoA/p38MAPK/NF-кB/F-Actin Signal Pathway}

\author{
Ran Deng ${ }^{1,2+}$, Feng $\mathrm{Li}^{1,2+}$, Hong Wu ${ }^{1,2 *}$, Wen-yu Wang ${ }^{1,2}$, Li Dai1,2, Zheng-rong Zhang ${ }^{1,2}$ \\ and Jun $\mathrm{Fu}^{1,2}$ \\ ${ }^{1}$ Key Laboratory of Xin'an Medicine, Ministry of Education, Hefei, China, ${ }^{2}$ College of Pharmacy, Anhui University of Chinese \\ Medicine, Hefei, China
}

Geniposide (GE) is the extraction and purification of iridoid glycosides from the Gardenia jasminoides Ellis, which is a promising anti-inflammatory drug, but its mechanism of actions on rheumatoid arthritis (RA) has not been clarified. This study investigated the molecular mechanism behind GE reduced the high permeability of fibroblast-like synoviocytes (FLSs) derived from SD rats with adjuvant arthritis (AA), with the aims of observing the action of GE in AA rats and exploring new therapeutic strategies for RA treatment. The CCK-8 method was used to detect FLSs proliferation. The pro-inflammatory cytokines levels and anti-inflammatory cytokines levels in FLSs were determined by ELISA kits. FLSs permeability assay was performed on Transwell. Immunofluorescence was used to assay the arrangement and morphology of F-actin. The expression of the key molecules related to FLSs permeability (RhoA, p-p38MAPK, NF-kB p-p65 and F-actin) was detected by western blotting. After treatment with lipopolysaccharide (LPS), the proliferation and the permeability of the cells increased significantly (all $P<0.05$ ). The expression of RhoA, p-p38MAPK, NF-кB p-p65 and F-actin in FLSs was higher compared with the control group, and F-actin was redistributed, with the formation of additional stress fibers. But, these conditions were moderated after treatment with GE. We demonstrated that the treatment of different concentrations of GE $(25,50$, and $100 \mu \mathrm{g} / \mathrm{mL})$ had a significant inhibitory effect on the proliferation and permeability of FLSs in vitro. Furthermore, the levels of interleukin (IL)-1 $\beta$ and IL-17 secreted by FLSs were decreased in different doses of GE groups, and the levels of anti-inflammatory cytokines (IL-4, TGF- $\beta 1$ ) were increased. Under treatment with GE, low expression of RhoA downregulated expression of p-p38MAPK, NF-кB p-p65, and F-actin while compared with control group, and restored the hyperpermeability of FLSs due to LPS treatment. Taken together, GE might play its anti-inflammatory and immunoregulatory effects via regulating the 
relative equilibrium of pro-inflammatory cytokines and anti-inflammatory cytokines. GE attenuated the hyperpermeability of FLSs. The down-regulation of the conduction of RhoA/p38MAPK/NF-kB/F-actin signal may play a critical role in the mechanisms of GE on RA. GE could be an effective therapeutic agent for the treatment of RA.

Keywords: geniposide, adjuvant arthritis, fibroblast-like synoviocytes, F-actin, perpermeability, inflammatory cytokines

\section{INTRODUCTION}

Rheumatoid arthritis (RA) is a systemic, progressive and chronic inflammatory immune disease (Firestein, 2003; Firestein and McInnes, 2017), which is characterized by synovial tissue inflammation, the formation of synovial pannus, and progressive joint damage of cartilage and bone (See et al., 2013; Mankia and Emery, 2015; Chang et al., 2015). Abnormal proliferation of fibroblast-like synoviocytes (FLSs) is a central event in the development of RA (Filer, 2013). FLSs show tumor cell-like growth. It can secrete large amounts of inflammatory cytokines, chemokines, matrix metalloproteinases (MMPs), regulate the formation of pannus and destruct cartilage and bone, eventually lead to joint deformity and disfunction. It is reported that pro-inflammatory cytokines are activated by multiple signaling pathways, such as the RhoA/ROCK, mitogen-activated protein kinases (MAPKs) and nuclear factor $\kappa \mathrm{B}$ (NF- $\kappa \mathrm{B}$ ) pathways (Xiao et al., 2013; Zhuang et al., 2017). Recent studies found that MAPKs, NF-кB, and Rho/ROCK pathways are highly activated in FLSs and involved in the pathogenesis of RA (Ganesan et al., 2016; Liu et al., 2017; Yokota et al., 2017). Blocking MAPKs, NF- $\kappa B$ and Rho/ROCK signaling pathways to find a new antiinflammatory drugs for RA treatment has been a focus.

Geniposide (GE), a kind of iridoid glycosides, derived from Gardenia jasminoides Ellis, with anti-inflammatory, antioxidation and anti-angiogenesis and other pharmacological effects (Fu et al., 2012; Xiaofeng et al., 2012). Our previous studies found that GE could control the activity of cytokines and decrease the levels of inflammatory medias in adjuvant arthritis (AA) rats (Wu et al., 2008). It can inhibit the proliferation of FLSs and decrease the levels of Interferon-gamma (IFN- $\gamma$ ), IL-6, IL-17 and other inflammatory cytokines levels and increase the level of anti-inflammatory cytokines such as TGF- $\beta 1$, IL-4 that secreted by FLSs or mesenteric lymph node (Dai et al., 2014; Wang R. et al., 2017). These effects were related to the amelioration of Jun N-terminal kinase (JNK) signaling and p38 signaling of MAPKs signaling cascade in FLSs (Chen J. et al., 2015). All of these studies indicated that GE may play a key role in regulating FLSs permeability. MAPKs signaling pathways are important for extracellular signals that cause fine internal reactions. In RA tissue, p38MAPK is activated in synovial lining and endothelial cells. It was found that p38MAPK was activated by inflammatory stimulant (LPS, IL-1, IL-6, etc.), mitogen, growth factor and so on. Activated p38MAPK could regulate the expressions of MMPs, NF-KB and fibrinous actin (F-actin), etc., which played an important role in the pathogenesis of RA joint swelling, synoviocyte dysplasia, angiogenesis, articular cartilage and bone erosion and progressive destruction (Li et al., 2013; Criado et al., 2014; Wang et al., 2015; Zou et al., 2016).
Cytoskeleton is a complex fibrous network composed of protein filaments in the cytoplasm. It mainly consists of three kinds of protein fibers: microtubules, microfilaments and intermediate fibers (Singer et al., 1985). Cytoskeleton plays an important role in maintaining cell morphology, such as cell survival, migration, apoptosis and the transport of substances inside and outside the membrane. Actin is a structural protein microfilament, mainly in both monomer and polymer form. Monomeric actin, also known as globular actin (G-actin) is composed of a polypeptide chain spherical molecules. F-actin filaments are actin filaments formed by actin multimers and are cytoskeletal fibers with a diameter of about $7 \mathrm{~nm}$ (Puthenedam et al., 2007). The actin cytoskeletal network plays a major role in the maintenance of the endothelial barrier function, as it regulates the integrity of the cells. In inflammatory cytokines, growth factors, poisoning, hypoxia, ischemia and other pathological conditions, those can cause F-actin intracellular morphological and distribution changes, lead to some physiological barrier permeability increased, thus impairing its function (Connolly et al., 2011). Ras superfamily related RhoA/Rho pathway exists in many organisms. The regulation of intracellular actin polymerization and depolymerization by the RhoA/Rho pathway is responsible for the "molecular switch" that in turn triggers the downstream kinase cascade to play a biological role. RhoA/ROCK pathway is an important regulator of cell morphology and F-actin. It cannot only maintain the cell morphology, but also affect the cell adhesion through the correlation with the extracellular matrix and extracellular matrix. It plays a crucial role in various physiological activities such as cell migration, growth, proliferation and apoptosis (Dasgupta et al., 2017; Wei et al., 2017).

The mechanism of FLSs hyperpermeability induced by inflammatory cytokines has been illuminated to a certain extent. RhoA/ROCK signaling pathway plays a key role in the regulation of different cells permeability and barrier function (Wang et al., 2012; Yin et al., 2016; Dong et al., 2017). The study found that F-actin was disorder in bone marrow mesenchymal stem cells (MSC) in patients with systemic lupus erythematosus (SLE) (Dong et al., 2015). Protein expression was significantly higher than the normal control group; the expression levels of RhoA and F-actin were overexpressed in the MH7A cell line induced by IL-1 $\beta(10 \mathrm{ng} / \mathrm{mL}$ ) in RA patients (Lv et al., 2015). The increase of the F-actin expression in many diseases has been confirmed (Puthenedam et al., 2007; Ng et al., 2015). Kawanami et al. (2011) found that ROCK-specific inhibitor Y-27632 inhibited the phosphorylation of p38MAPK and p65, which indicated that ROCK was able to regulate $\mathrm{p} 38 \mathrm{MAPK} / \mathrm{NF}-\kappa \mathrm{B}$ signaling pathway. Thus, the intervention of F-actin by RhoA through 
p38MAPK/NF- $\mathrm{B}$ signaling pathway may be a new target for RA therapy.

In this study, FLSs isolated from AA rats have been used to determine the effectiveness of GE. Furthermore, we used LPS-treated FLSs, observed the influence of GE on FLSs hyperpermeability and assessed the effect of GE on the RhoA/p38MAP/NF- $\kappa$ B/F-actin signaling pathway and then constructed a new therapeutic effect target.

\section{MATERIALS AND METHODS}

\section{Materials and Reagents}

Geniposide was extracted and purified from G. jasminoides Ellis (purity $>98 \%$ ), and provided by the National Institute for the Control of Pharmaceutical and Biological Products (Beijing, China). Fetal bovine serum (FBS) was obtained from HyClone (Logan, United States). Rabbit monoclonal antibodies against p38, p-p38, NF- $\kappa$ B p65 and NF- $\kappa$ B p65 were supplied from Cell Signaling Technology (Beverly, MA, United States). AntiRhoA antibody was purchased from Abcam (Cambridge, MA, United States). $\beta$-actin and HRP-conjugated goat anti-rabbit antibodies were provided by ZSGB-BIO (Beijing, China). Rhokinase inhibitor (Y-27632) was obtained from Selleck (Houston, TX, United States). Rat IL-4, IL-1 $\beta$, IL-17 and TGF- $\beta$ ELISA kits were purchased from Elabscience Biotechnology (Wuhan, China). Freund's Complete Adjuvant (FCA) and LPS were supplied from Sigma Chemical Company (St. Louis, MO, United States). Other chemicals that have been used in this work were of research grade.

\section{Animals}

Male Sprague-Dawley (SD) rats $(200 \pm 20$ g, Grade II, Certificate NO.078) were purchased from the Experimental Animal Center of Anhui University of Chinese Medicine (Hefei, China). All rats were housed under specific pathogen-free conditions with a 12 -h light/dark cycle in a temperature-controlled room at $25 \pm 1^{\circ} \mathrm{C}$ and $50-60 \%$ relative humidity. All rats were maintained in this condition at least 7 days before experiment. All studies on rats were carried out following the Guideline for Animal Experiments of Anhui University of Chinese Medicine and the protocol was approved by the Ethics Review Committee for Animal Experimentation of Anhui University of Chinese Medicine.

\section{Induction and Evaluation of Adjuvant Arthritis Rats}

Adjuvant arthritis models were induced according to the previous method. Rats were immunized on day 0 by a single intradermal injection into the right hind paw with $100 \mu \mathrm{L}$ of FCA, while the normal group rats were given the same volume of physiological saline at the same time $(n=12)$. Rats were randomly divided into two groups: normal control group, AA model group, 6 rats in each group. At the day 7 , day 11 , day 14 , day 17 , day 21 , two groups of rats were evaluated from the paw volume, arthritis index and arthritis systemic assessment. All the rats were examined for signs of arthritis by two independent and blind to the experimental design of observers. Non-injected hind paws volume of rats were measured with PV-200 volume meter (Chengdu Taimeng Technology Co., Ltd., Chengdu, China). The degree of secondary joint swelling in rats was calculated as ( $\Delta \mathrm{mL}=$ volume of the foot after modeling - the volume of the foot before modeling).

The arthritis systemic assessment was scored according to the degree of swelling of the fore foot and posterior foot of the rat and the presence of nodules and erythema in the nasal, ear and tail. The specific standard is as follows: Nose: $0=$ no connective tissue swelling, 1 = significant connective tissue swelling; Ears: $0=$ No nodules and redness, $1=$ nodules and redness appear in one ear, 2 nodules and redness appear in both ears; Tail: $0=$ no nodules, 1 = nodules; Forefoot: 0 = no swelling, 1 = paw swelling in one forefoot, 2 = paw swelling in both forepaws; Hind paw: $0=$ no swelling, $1=$ swelling of one hind paw, $2=$ swelling of both hindpaws. Each rat scored up to 8 points.

The arthritic index was based on the occurrence and severity of secondary lesions of arthritis in rats. Grading standards are evaluated as follows: $0=$ no redness; 1 = redness of the foot metatarsal joint; 2 = redness of the toe joint and toes; $3=$ redness below the ankle joint; 4 = redness of the entire foot, including the ankle joint. Each rat has a maximum of 12 points.

\section{Preparation and Culture of FLSs}

Fibroblast-like synoviocytes from AA rats synovial tissues were isolated by the method of tissue explant cultivation as described previously (Wei et al., 1986). AA rats were anesthetized and sacrificed by bleeding from the abdominal aorta on day 21 after immunization. The fresh synovial tissue were taken out in sterile condition and put into culture dishes with D-Hank's solution, washed and removed of all fat and connective tissue. Then, AA rats synovial tissues were cut into small pieces of $1-2 \mathrm{~mm}^{3}$, incubated in a flat bottom culture bottle and cultured in DMEM supplemented with $20 \% \mathrm{FBS}$ at $37^{\circ} \mathrm{C}$ and $5 \% \mathrm{CO}_{2}$. The culture solution was changed every 2-3 days. When a large number of FLSs grew from the synovial tissue, small pieces of tissue were discarded. Adherent cells were trypsinized, cells were routinely split at a 1:2/1:3 ratio, and cultured in medium. According to the growth of FLSs and the change of the color of the culture medium, the fluid was changed once every 1-2 days. The FLSs obtained from the 3 th to 4 th passages were used for additional experiments. Flow cytometry analysis was used to identify the purity of FLSs according to the expression of vascular cell adhesion molecule-1 (VCAM-1). The results indicated that the expression of VCAM-1 in passaged third synovial cells was $99.5 \%$ (Figure 1). The results showed that the third generation of cell suspension cultured mainly FLSs.

\section{Proliferation Assay by CCK-8}

Fibroblast-like synoviocytes obtained from AA rats were planted into 96-well plate at a concentration of $1 \times 10^{5}$ cells $/ \mathrm{mL}$ containing $100 \mu \mathrm{L}$ DMEM medium with $10 \% \mathrm{FBS}$ at $37^{\circ} \mathrm{C}$ for $24 \mathrm{~h}$ in a $5 \% \mathrm{CO}_{2}$ incubator. The FLSs were divided into control group, AA group, different doses of GE groups $(6.25,12.5,25$, 50 , and $100 \mu \mathrm{g} / \mathrm{mL}$ ) and Y-27632 inhibitor group. The cells were incubated at $37^{\circ} \mathrm{C}$ for $48 \mathrm{~h}$ in a $5 \% \mathrm{CO}_{2}$ incubator. Subsequently, 

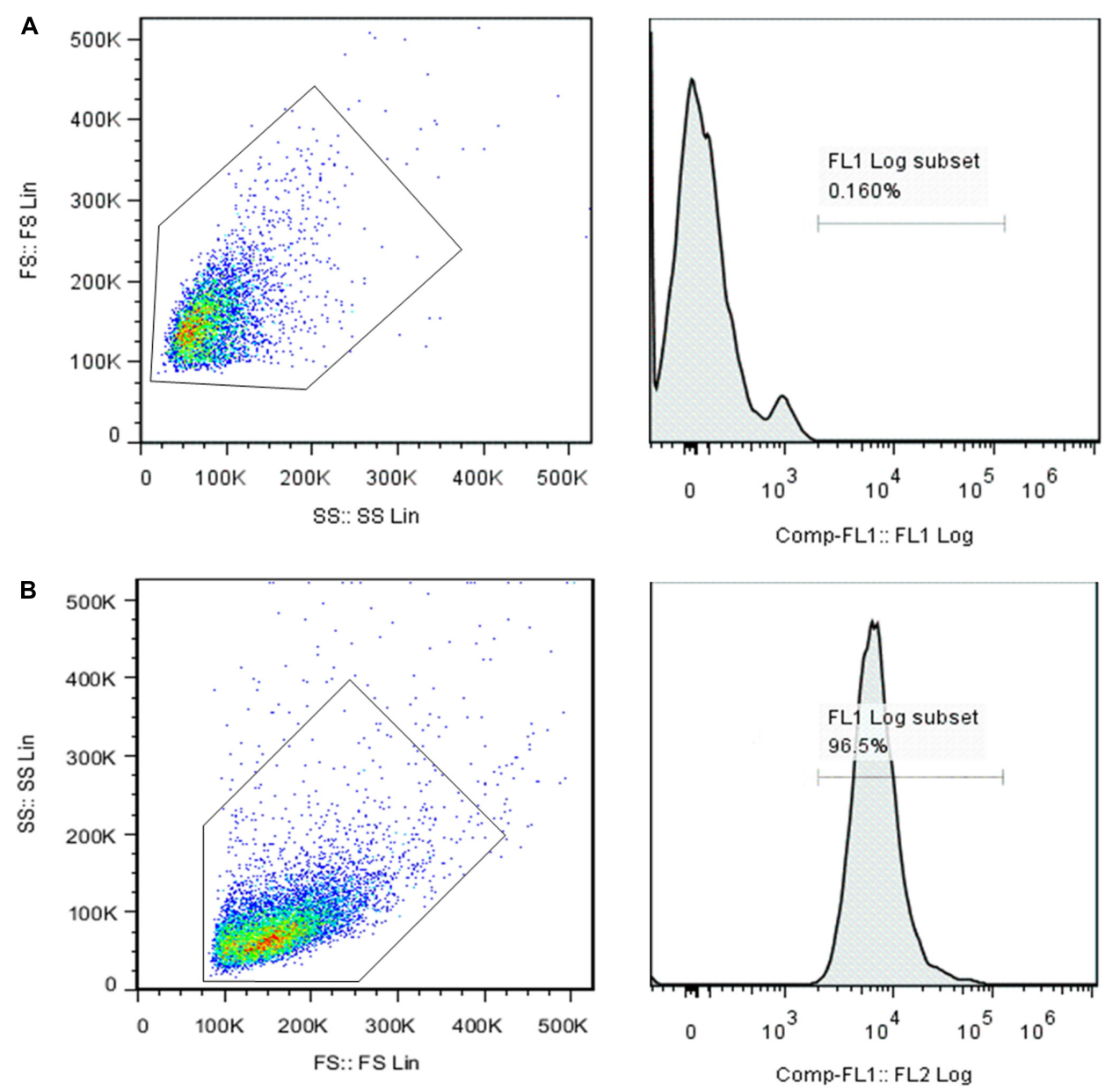

FIGURE 1 | Flow cytometry analysis of the surface maker of the FLSs. Mouse lgG isotypes labeled with FITC served as a negative control (A). The positive rate of $0.16 \%$ was chosen as the background (as shown in the box) and then the FITC-labeled VCAM-1 (B) expression rate was examined. The results showed that the expression of VCAM-1 in the cell suspension was 99.5\%, indicating that the main component of cultured third-generation synoviocytes was FLSs.

the cells were washed with PBS twice. Then, $10 \mu \mathrm{L}$ CCK-8 (Japanese colleagues Chemical, Japan) was added to each well, and cells were further incubated for another $4 \mathrm{~h}$. The optical density values of each well were examined at $450 \mathrm{~nm}$ using a microplate reader (Haiou Pei Analytical Instruments Co., Ltd., China, Shanghai).

\section{FLSs Monolayer Permeability/Barrier Function}

Bovine serum albumin (BSA) diffusion through FLSs monolayers was evaluated using transwell cell culture chambers (collagencoated polycarbonate filters with a pore size of $0.4 \mu \mathrm{m})$. A cell suspension $\left(1 \times 10^{5}\right.$ cells $)$ was added to the upper chambers of the Transwell apparatus. FLSs seeded in the upper chambers of transwells were maintained in culture for a period of 12 days to obtain monolayers, with culture media being changed on alternate days. To measure monolayer barrier function, cell culture medium was replaced with media containing BSA (200 mg/L), before initiating drug treatment for $0.5 \mathrm{~h}$ at $37^{\circ} \mathrm{C}$.
Then, the cells were divided into six groups ( $n=3$ transwells): control group, AA group, GE groups $(25,50$, and $100 \mu \mathrm{g} / \mathrm{mL})$, Y-27632 $(10 \mu \mathrm{M})$. After $48 \mathrm{~h}$ of drug treatment, media from the lower chamber were collected to monitor BSA content by Bradford method.

\section{The Induction and Activity of IL-1 $\beta$, IL-17, IL-4, and TGF- $\beta 1$ (Talaat et al., 2015)}

The FLSs of AA rats were planted into six-well plates at a density of $2 \times 10^{5}$ cells/well at $37^{\circ} \mathrm{C}$ in a $5 \% \mathrm{CO}_{2}$ incubator. Then, the cells were divided into six groups: control group, AA group, GE groups (25, 50, and $100 \mu \mathrm{g} / \mathrm{mL})$, Y-27632 $(10 \mu \mathrm{M})$. The supernatants were collected to measure the levels of IL- $1 \beta$, IL-17, IL- 4 , and TGF- $\beta 1$. The cytokine levels in the culture supernatants of FLSs were determined by using murine or rat specific ELISA kits for IL-1 $\beta$, IL-17, IL-4, and TGF- $\beta 1$ according to the manufacturer's instructions (Elabscience Biotechnology, Wuhan, China). 

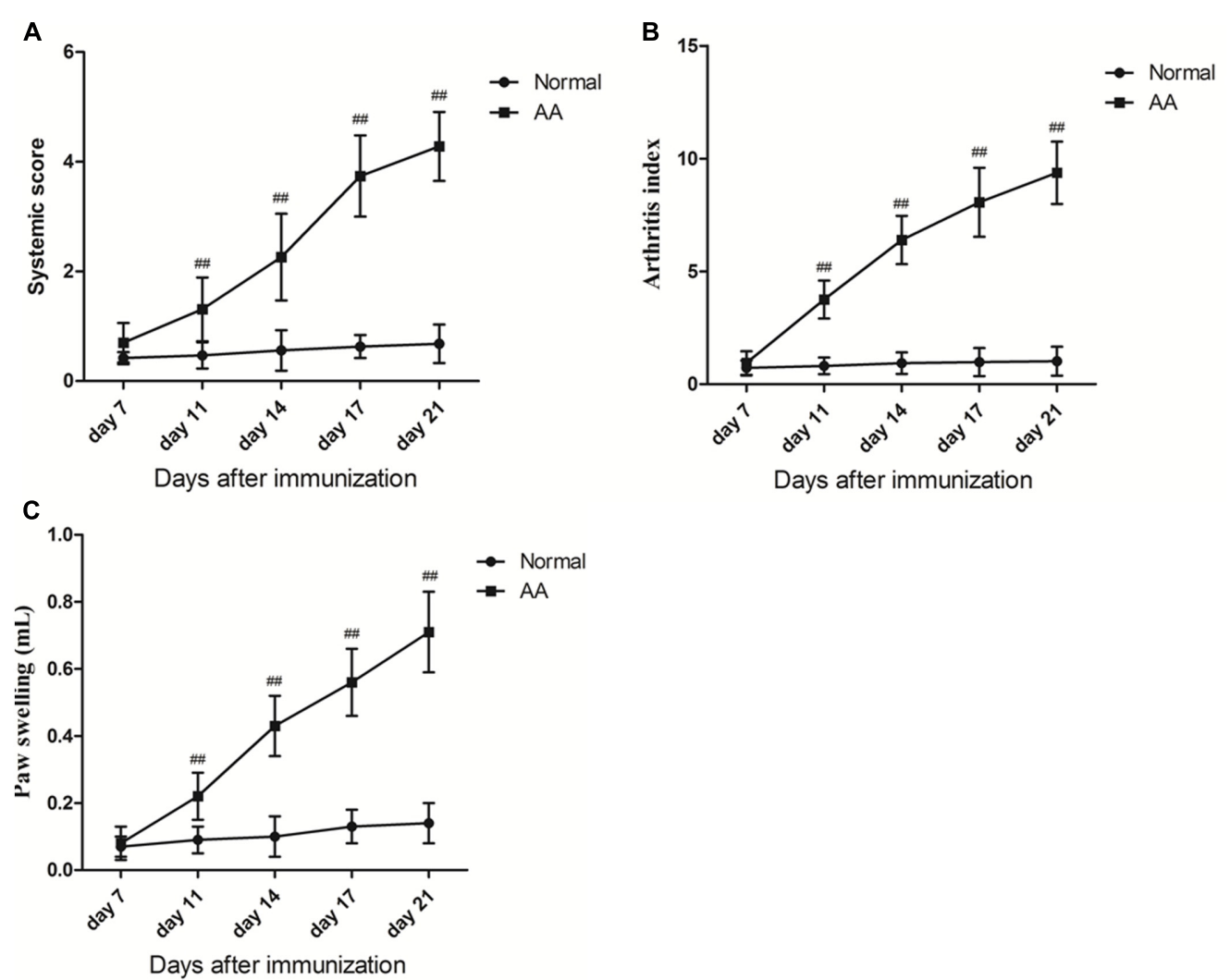

FIGURE 2 | (A) The systemic score assessment of normal group and AA group rats $(x \pm s, n=6)$. (B) The inflammatory poly arthritis index score of normal group and AA group rats $(x \pm s, n=6)$. (C) Secondary normal group and AA group rats foot swelling of change $(\Delta \mathrm{mL})(x \pm s, n=6)$. Rats were immunized on day 0 by a single intradermal injection into the right hind paw with $100 \mu \mathrm{L}$ of FCA. At the day 7 , day 11 , day 14 , day 17 , day 21 , two groups of rats were evaluated from the paw volume, arthritis index and arthritis systemic assessment. Data are presented as means $\pm \mathrm{SD}$. \#\# $P<0.01$ vs. normal group.

\section{Immunofluorescence Staining}

To further observe changes of F-actin in FLSs, immunofluorescent staining was performed. FLSs were detached with $0.25 \%$ Trypsin-EDTA. Cultured FLSs under different conditions were plated onto different 24-well plates and fixed in $4 \%$ paraformaldehyde for $10 \mathrm{~min}$, followed by $1 \%$ BSA (with $0.3 \%$ Triton $\mathrm{X}-100$ ) for $1 \mathrm{~h}$ at room temperature. The F-actin of FLSs were incubated with $200 \mu \mathrm{L}$ FITC-conjugated phalloidin (150 nM) (Sigma). DAPI was used to visualize the nuclei at room temperature for $1 \mathrm{~h}$. Samples were examined using a confocal laser scanning microscope.

\section{Western Blotting Analysis}

The standard method was used to analysis of the proteins extracted from FLSs by Western blot. The protein concentration was assayed by BCA Protein Assay Kit. The proteins were separated by $10 \%$ sodium dodecyl-sulfate-polyacrylamide gel electrophoresis (SDS-PAGE) and were electrophoretically transferred to nitrocellulose (NC) membranes (Millipore, Bedford, MA, United States). After blockage with 5\% skim milk at room temperature for $2 \mathrm{~h}$, the membranes were incubated with the specific primary antibodies against $\beta$-actin, RhoA, p38, p-p38, p65, p-p65, and F-actin at $4^{\circ} \mathrm{C}$ overnight on a rotary shaker.
The membranes were washed in Tris-buffered saline/Tween 20 (TBST) and then incubated horse radish peroxidase conjugated secondary antibody for $2 \mathrm{~h}$. The membranes were washed in TBST again and the immunoreactive proteins were detected with Pierce $^{\mathrm{TM}}$ ECL Western Blotting Substrate (Thermo Scientific, MA, United States). The protein bands were scanned by Alpha View SA system (ProteinSimple, San Jose, CA, United States) and analyzed using Image software.

\section{Statistical Analysis}

All data were expressed as mean \pm standard deviation (SD). The Statistical analysis was performed by SPSS 21.0 software, and the differences between groups were carried out by Student's $t$-test and One-way ANOVA test. Value of $P<0.05$ or $P<0.01$ was considered statistically significant.

\section{RESULTS}

\section{Evaluation of Arthritis on AA Rats}

To extract FLSs primary cells from AA rats, the AA models in SD mice were employed. After healthy SD rats were immunized with FCA for the first time, $\mathrm{d} 1-\mathrm{d} 3$ showed redness in the right 
side of the rat, which was caused by subcutaneous injection of inflammation. On the 7th day after immunization, the primary swelling of right hind paws in AA rats was reappeared. On day 11 after immunization, the secondary swelling of left hind paws in AA rats arose obvious secondary inflammatory response, which showed paw swelling, pain, polyarthritis, nose, ear and tail appeared nodules and erythema. The systemic score assessment, polyarthritis index, and right hind paw swelling of AA rats were significantly increased compared with normal rats $(P<0.01)$. The results were shown in Figure 2. In this study, typical and stable AA rats were successfully established.

\section{GE Inhibited the Proliferation of FLSs in AA Rats}

As shown in Figure 3, the study results of CCK-8 suggested that the optimal concentration of GE were 25,50 , and $100 \mu \mathrm{g} / \mathrm{mL}$ for FLSs proliferation. Thus, 25, 50, and $100 \mu \mathrm{g} / \mathrm{mL}$ were chosen as the appropriate concentrations of GE in the next test. The proliferation activity of FLSs in AA group enhanced significantly compared to control group $(P<0.01)$. Compared with AA model group, GE groups $(25,50$, and $100 \mu \mathrm{g} / \mathrm{mL})$ had a significant inhibitory effect on LPS-induced FLSs cell proliferation $(P<0.01)$, Y-27632 inhibitor group $(10 \mu \mathrm{M})$ also showed significantly inhibitory on proliferation of FLSs which maybe related RohA/ROCK pathway $(P<0.01)$.

\section{GE Relieved LPS-Induced Hyperpermeability of FLSs}

Using monolayers of FLSs in a Transwell apparatus, we tested whether GE can directly affect the high permeability of FLSs induced by LPS via evaluating the permeability to BSA. As seen in Figure 4, the results showed that LPS significantly increased the permeability of FLSs, indicating the formation of hyperpermeability of FLSs. In GE groups $(25,50$, and $100 \mu \mathrm{g} / \mathrm{mL})$ $(n=3)$, transport of BSA across the FLSs was decreased following the addition of GE compared to the untreated LPS group and exhibited a dose-dependent manner. And FLSs permeability was positively correlated with the relative concentration of BSA in the lower chambers of transwell medium of each group.

\section{Effects of GE on Cytokines Secretion of FLSs}

To confirm the anti-inflammatory effects in vitro, GE was evaluated for its inhibition of pro-inflammatory (IL-1 $\beta$ and IL-17) mediators and increase of anti-inflammatory (IL-4 and TGF- $\beta 1$ ) in FLSs stimulated by LPS. The result was shown in Figure 5. The results of ELISA showed that the levels of IL-1 $\beta$ and IL-17 in FLSs were significantly up-regulated compared with the normal group, and the levels of antiinflammatory cytokines IL-4 and TGF- $\beta 1$ was significantly declined $(P<0.01)$. Compared with AA model group, different doses of GE groups $(25,50$, and $100 \mu \mathrm{g} / \mathrm{mL})$ could significantly decrease the secretion of IL-1 $\beta$ and IL-17 in FLSs, and significantly increase the secretion of IL- 4 and TGF- $\beta 1$ $(P<0.01)$. The levels of IL-1 $\beta$ and IL-17 in FLSs were
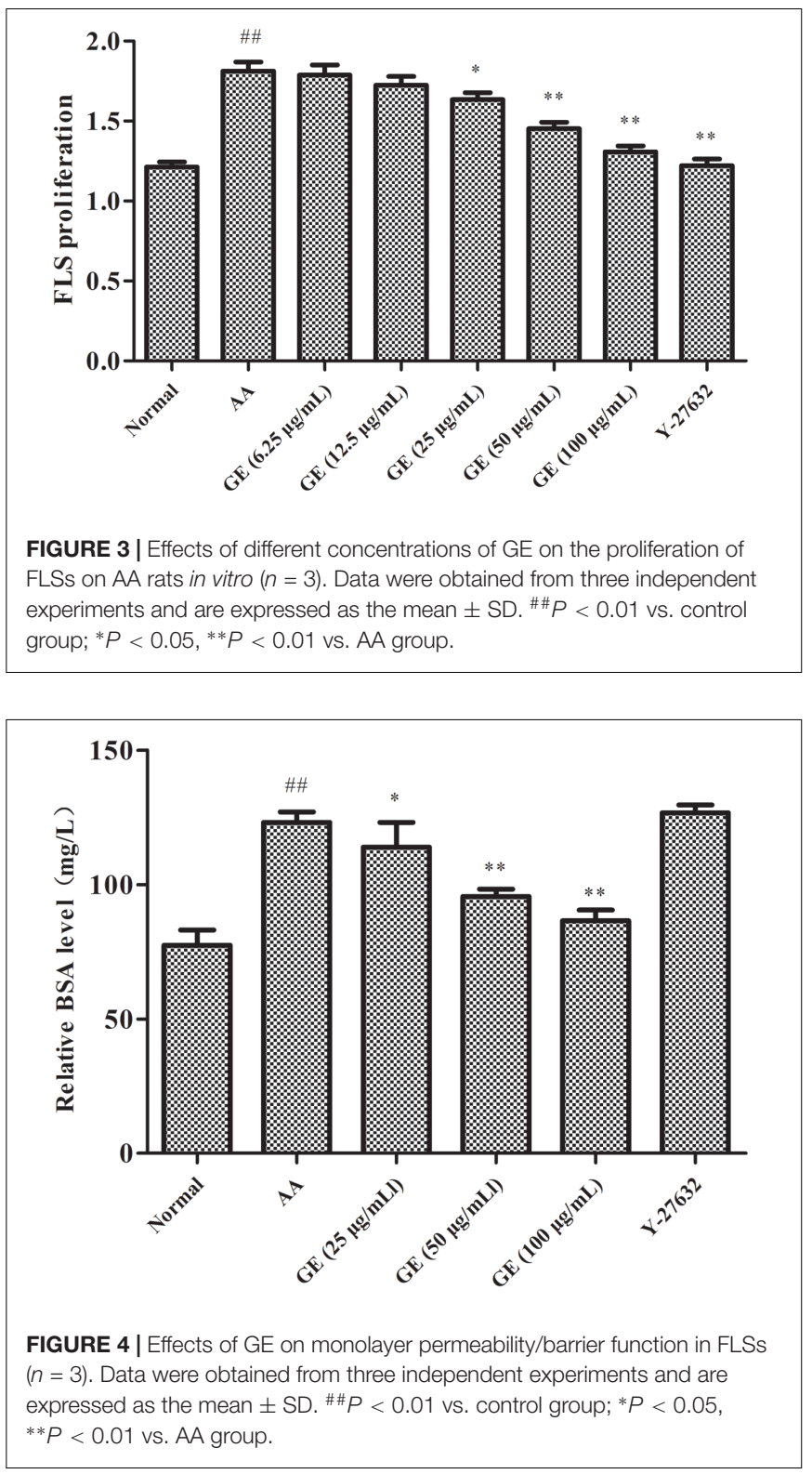

significantly decreased while treatment with Y-27632 inhibitor, and the levels of IL- 4 and TGF- $\beta 1$ were significantly increased $(P<0.01)$.

\section{GE Affected the Distribution of F-Actin in FLSs Induced by LPS}

In order to observe changes in the cytoskeleton, immunofluorescence was used to observe F-actin in FLSs. It could be observed in Figure 6 that under confocal laser scanning microscope, FITC-phalloidin labeled F-actin showed green fluorescence and DAPI-labeled nuclei showed blue fluorescence. We observed that F-actin is mainly located in the outer periphery of the cell and inside the cell membrane, with a complete cell membrane and clear cell borders. In contrast, when FLSs were incubated with LPS, cell boundaries 


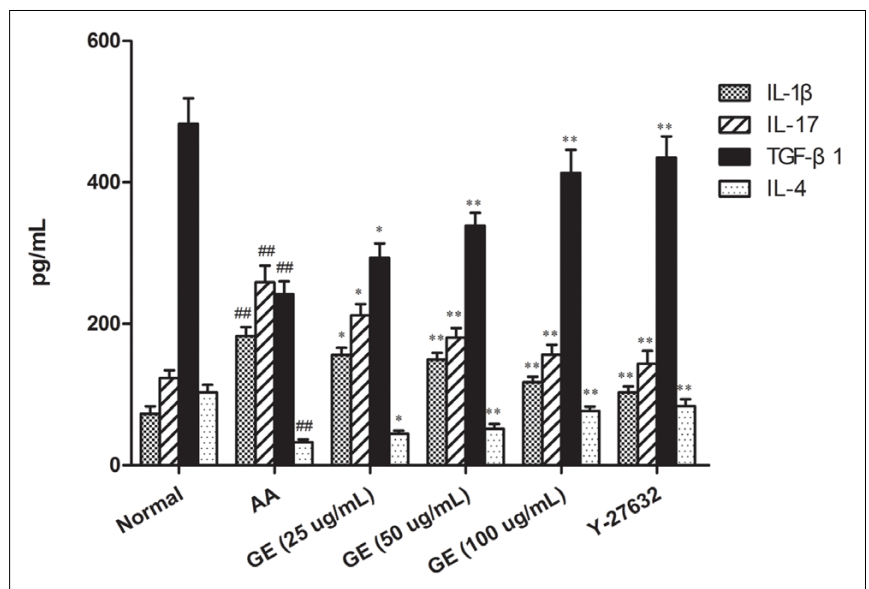

FIGURE 5 | Effects of GE on production of IL-1 $\beta$, IL-17, TGF- $\beta 1$, and IL-4 in FLSs of AA rats. The cell supernatants were collected to detect the levels of $I L-1, I L-17, T G F-\beta 1$, and IL- 4 by ELISA $(n=3)$. Data were obtained from three independent experiments and are expressed as the mean $\pm \mathrm{SD}$. ${ }^{\#} P<0.01$ vs. control group; $* P<0.05, * * P<0.01$ vs. AA group.

became obscured and dense F-actin stress fibers accumulated. FLSs also induced an increase in F-actin, as determined by western blot compared with normal group. The doses of GE (25, 50, and $100 \mu \mathrm{g} / \mathrm{mL}$ ) could reduce F-actin fibers in FLSs cytoplasm in different degrees, and F-actin fibers accumulated on the periphery of the membrane. Moreover, when FLSs pretreated with Y27632, the location and the amount of F-actin resembled that of unstimulated cells, thus indicating that this pathway may be responsible for AA-induced cytoskeletal rearrangement.

\section{GE Inhibited the High Expression of RhoA, p-p38MAPK, NF-kB p-p65, and F-Actin Protein of FLSs Induced by LPS}

We have already identified AA did enhance FLSs permeability, then we wish to determine whether AA induced permeability increase via this pathway. First, in order to confirm whether AA induced activation of RhoA/p38MAPK/NF- $\mathrm{BB} / \mathrm{F}$-actin pathway in FLSs, The phosphorylation of RhoA, p-p38MAPK, NF- $\mathrm{B}$ $\mathrm{p}-\mathrm{p} 65$, and F-actin was identified. The experimental results of protein electrophoresis were shown in Figure 7. The expression of RhoA, p-p38MAPK, NF- $\mathrm{B}$ p-p65, and F-actin of FLSs in AA model group was significantly upregulated while compared with normal group $(P<0.01)$. The results demonstrated that RhoA/p38MAPK/NF-KB/F-actin pathway are highly activated in FLSs by LPS. Different doses of GE groups $(25,50$, and $100 \mu \mathrm{g} / \mathrm{mL}$ ) significantly reduced RhoA, p-p38MAPK expression and $\mathrm{F}$-actin protein in FLSs $(P<0.01)$. There was significant difference in the expression level of NF- $\kappa$ B p-p65 protein $(P<0.01)$ only in the middle and high dose of GE groups (50 and $100 \mu \mathrm{g} / \mathrm{mL}$ ). The levels of RhoA, p-p38MAPK, NF-kB p-p65 and F-actin proteins in FLSs was also significantly reduced by $\mathrm{Y}-27632$ inhibitor group $(10 \mu \mathrm{M})$ $(P<0.01)$.

\section{DISCUSSION}

Rheumatoid arthritis is a classic systemic autoimmune disease characterized by persistent chronic synovitis, vascularization, further destruction of articular cartilage and bone, and even deformity and serious complications (Walsh et al., 2010; Kong et al., 2016). Synovial cells are the major effector cells during RA. FCA induced AA model is a chronic, systemic, autoimmune inflammation mediated by $\mathrm{T}$ cells. It is very similar to human RA in histopathological and laboratory parameters and is ideal models for screening and studying drugs for treating RA (Chen C.X et al., 2015; Qian et al., 2017). AA rats mainly manifested as multiple peripheral arthritis and local joint swelling, deformity, pathological changes of proliferative synovitis, articular cartilage damage, bone erosion, inflammatory cell infiltration. And AA synovial also appeared neovascularization phenomenon, which is similar to human RA. Therefore, the AA rat model is an ideal model for screening and studying RA therapies.

Fibroblast-like synoviocytes play an important role in maintaining the homeostasis of the joint cavity in the normal body. In RA, a large number of harmful substances (inflammatory cytokines, inflammatory mediators, etc.) gathered in the joint cavity, abnormal proliferation and secretion of synovial cells, eventually leaded to articular cartilage and bone destruction (Byng-Maddick and Ehrenstein, 2015; Firestein and McInnes, 2017). Abnormal changes in rheumatoid arthritis synovial cells (RASFs) play a major role in the pathogenesis of RA. In the normal body, synovial cells control the structural and dynamic integrity of the joint movement by controlling the synovial fluid and the composition of the extracellular matrix in the joint lining. However, RASFs exhibit surprising pathogenic behavior, which increase in number, destroy vasoconstrictions, and have a serious effect on the induction of RA inflammation and joint damage. In this study, the proliferation of FLSs in AA rats induced by LPS was significantly faster than that in normal controls, while different concentrations of GE $(25,50$, and $100 \mu \mathrm{g} / \mathrm{mL})$ and $\mathrm{Y}-27632$ inhibitor $(10 \mu \mathrm{M})$ could significantly inhibit the proliferation of FLSs, suggesting that GE has an inhibitory effect on abnormal proliferation of FLSs obtained from AA rats.

Rheumatoid arthritis is a typical progressive, systemic inflammatory disease, whose major pathologic changes are associated with lymphocyte, synoviocyte synthesis of inflammatory cytokines and their receptors associated with abnormal gene expressions. The immune dysfunction is considered to be the primary pathogenesis of RA (Chakera et al., 2012; Lubberts, 2015; Tan et al., 2017). In the normal body, anti-inflammatory and pro-inflammatory cytokines maintain a dynamic balance, but this balance is broken in RA. IL-1 $\beta$ and IL-17 cannot only induce the proliferation of FLSs, but also stimulate the angiogenesis by promoting the expression of vascular endothelial growth factor (VEGF), IL-8, MPPs and other forms of angiogenesis, leading to the formation of pannus and synovial lesions of RA (Kim et al., 2017; Wang Y. et al., 2017). IL-4 is an important anti-inflammatory cytokines, which can enhance neutrophil-mediated phagocytosis and cytotoxicity and play an important role in maintaining humoral immunity 


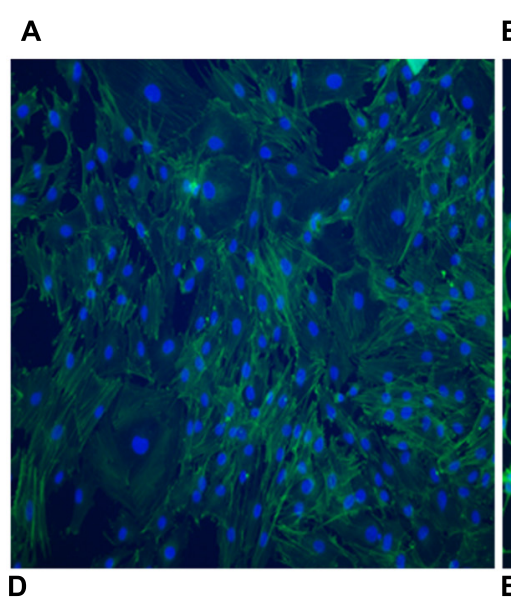

B $\quad$ C
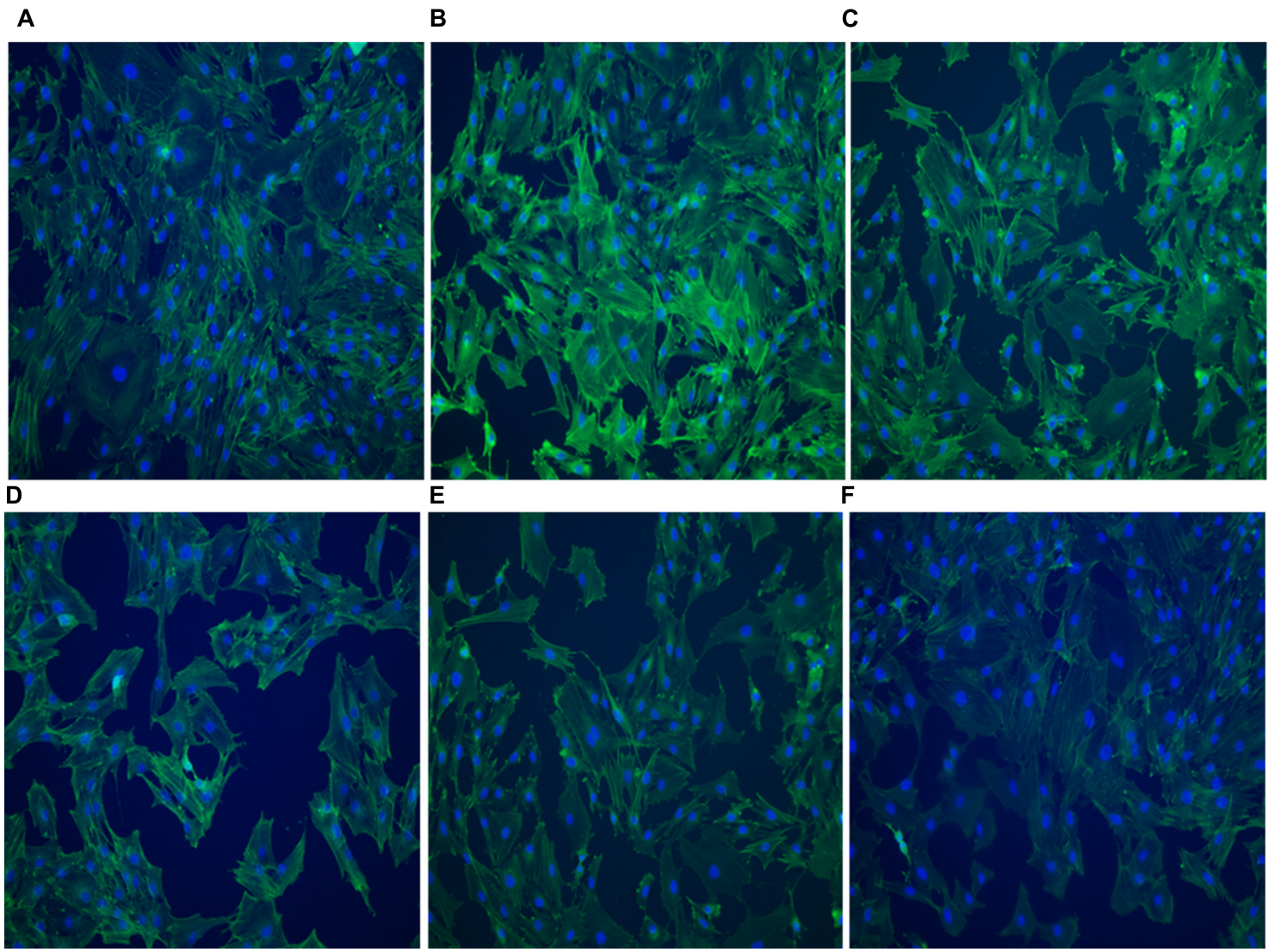

FIGURE 6 | Immunofluorescence images of F-actin of FLSs in AA rats $(n=3)(200 \times)$. FITC-phalloidin labeled F-actin showed green fluorescence and DAPI-labeled nuclei showed blue fluorescence. (A) Normal group, (B) AA group, (C) GE $(25 \mu \mathrm{g} / \mathrm{mL})$ group, (D) GE $(50 \mu \mathrm{g} / \mathrm{mL})$ group, (E) GE (100 $\mu \mathrm{g} / \mathrm{mL}) \mathrm{group}$, (F) Y-27632 inhibitor group $(10 \mu \mathrm{M})$.

in vivo (Krabben et al., 2013). TGF- $\beta 1$ is an anti-inflammatory cytokine that promotes the growth of fibroblasts and osteoblasts, and the expression of extracellular matrix such as collagen and fibronectin, thereby inhibiting inflammation and autoimmune responses (Suzuki et al., 2015). The balance was skewed toward pro-inflammatory cytokines, resulting in excessive production of IL-17 and IL-1 $\beta$, whereas treg activity and the level of TGF- $\beta 1$ were deficient in RA patients compared to healthy individuals. In this study, the levels of IL- $1 \beta$, IL-17, IL-4, and TGF- $\beta 1$ were evaluated to investigate whether the imbalance of pro-inflammatory cytokines and anti-inflammatory cytokines was involved in the inflammatory injury. The levels of proinflammatory cytokines IL-1 $\beta$ and IL-17 increased significantly in AA rats, while the levels of anti-inflammatory cytokines IL- 4 and TGF- $\beta 1$ decreased significantly, suggesting that the inflammatory symptoms were related to the expression levels of IL- $1 \beta$, IL-4, IL-17, and TGF- $\beta 1$ in FLSs. The levels of IL-1 $\beta$ and IL-17 in FLSs were significantly inhibited while the levels of IL- 4 and TGF- $\beta 1$ were up-regulated in different doses of GE groups $(25,50$, and $100 \mu \mathrm{g} / \mathrm{mL})$. While treatment with Y- 27632 inhibitor, the effect was similar to that of GE. These results suggested that inhibition of the levels of inflammatory cytokines, upregulation of anti-inflammatory cytokines, recovery of the dynamic balance between pro-inflammatory cytokines and anti-inflammatory cytokines are an important characteristic of GE regulation of immune balance.

Cytoskeleton is the basis for maintaining cell traits and positions, including microtubules, microfilaments and intermediate fibers. Actin is a structural protein of microfilaments in the forms of G-actin and F-actin. Under various stimuli, G-actin is polymerized into $\mathrm{F}$-actin with a highly dynamic structure. F-actin is unstable and has the function of disaggregation. F-actin polymerization and depolymerization between the state of conversion, that is, F-actin recombination, not only to maintain cell traits and location, but also mediate intercellular connections (such as: close connection, adhesion link, fissure link). The integrity of F-actin is essential for maintaining the normal function of cells. F-actin is a major cytoskeletal contraction protein. The increase of F-actin content can increase polymerization and form stress fibers, resulting in the cell tight junction structure cannot be maintained, thus increasing the permeability of monolayer, resulting in impairment of barrier function. We showed that LPS induced actin destabilization, which led to the hyperpermeability of FLSs in turn. The present results revealed that morphologically, GE attenuated LPS-induced stress fiber 

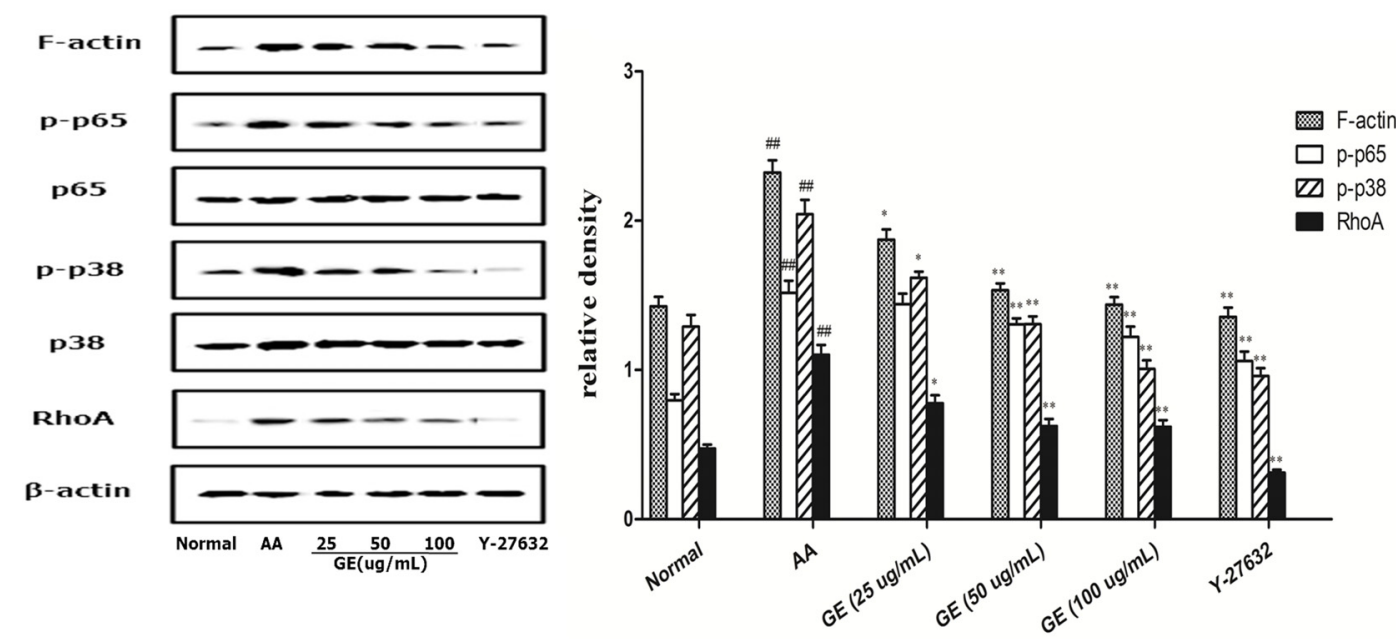

FIGURE 7 | Effects of GE on expression of RhoA, p38MAPK, NF-кB and F-actin in FLSs of AA rats. Western blotting analysis was performed to detect the expression levels of RhoA, p-p38MAPK, NF-kB p-p65 and F-actin $(n=3)$. The results were expressed as means \pm SD from three independent experiments. ${ }^{\# \#} P<0.01$ vs. control group; ${ }^{*} P<0.05,{ }^{* *} P<0.01$ vs. AA group. a: normal group, b: AA group, c: GE (25 $\left.\mu \mathrm{g} / \mathrm{mL}\right) \mathrm{group}, \mathrm{d}: \mathrm{GE}(50 \mu \mathrm{g} / \mathrm{mL}) \mathrm{group}, \mathrm{e}: \mathrm{GE}$ $(100 \mu \mathrm{g} / \mathrm{mL})$ group, f: Y-27632 inhibitor group (10 $\mu \mathrm{M})$.

formation by F-actin, which further confirmed that GE has an inhibitory effect on the LPS-induced hyperpermeability of FLSs.

Recent studies proved that the p38MAPK, Rho/ROCK pathways (Monickaraj et al., 2016; Ni et al., 2017) are involved in the regulation of microfilament assembly or contractility, thereby resulting in increased permeability. Studies found that thrombin and other stimulant can increase the F-actin polymerization to form stress fibers, and destroy the tight connection of cells, cause blood - joint capsule fluid barrier and other physiological barrier permeability increase, result in or aggravate the condition (Poli et al., 2002). Therefore, intervention of F-actin recombination may become a new target for RA treatment. Studies have shown that RhoA regulates actin polymerization/depolymerization through the Rho/ROCK pathway, causing actin recombination (Stanley et al., 2014). Activation of p38MAPK and NF-кB is an important cause of $\mathrm{F}$-actin depolymerization and recombination ( $\mathrm{Yu}$ et al., 2016). It was found that F-actin recombination and cell permeability were increased in pulmonary microvascular endothelial cells (PMVEC) induced by influenza virus, and the phosphorylation level of Ezrin/radixin/moesin (ERM) was up-regulated. ERM is the connection between the cell membrane and the actin cytoskeleton. Rho/ROCK inhibitor Y27632, p38MAPK inhibitor SB203580 can down-regulate ERM phosphorylation levels, inhibit F-actin recombination, and improve PMVEC cell permeability (Zhang et al., 2014). Thus, RhoA/p38MAPK/NF-кB/F-actin signaling pathway all contributes to hyperpermeability in cells via regulating F-actin. The current result is consistent with the previous report. The present results further demonstrated that the RhoA/p38MAPK/NF- $\kappa \mathrm{B} / \mathrm{F}$-actin signaling pathway is activated in response to LPS and caused F-actin rearrangement, which in turn mediated hyperpermeability of FLSs.
Geniposide alleviated hyperpermeability of the FLSs in vitro. Some studies have reported that GE has a therapeutic effect on RA. For instance, GE downregulated expression of TNF- $\alpha$, IL-2 and upregulated IL-1 $\beta$, IL-4, TGF- $\beta 1$ (Pan et al., 2017; Zhang et al., 2017). GE also has protective effects on LPSinduced endothelial barrier dysfunction (Xu et al., 2017). GE suppresses the release of inflammatory mediators, thus moderating the systemic inflammatory response. There have been no previous studies reporting direct effects of GE on FLSs permeability under inflammatory conditions. This study provided the first evidence to confirmed that GE directly modulated the stability of the permeability of the FLSs when exposed to the inflammation mediator and LPS. Moreover, our study confirmed that GE could regulate the inflammatory response in RA. This is GE's new mechanism for treating RA. We found that the molecular mechanism behind GE moderation of the permeability disorder of FLSs induced by LPS is via the RohA/p38MAPK/NF- $\kappa$ B/F-actin signal pathway. After stimulation by LPS, the expression of RhoA, p-p38MAPK, NF- $\kappa$ B p -p65 and F-actin increased significantly in AA group. It demonstrated LPS activated RhoA/p38MAPK/NF-кB/F-actin signaling pathway and subsequently led to reassembly of actin, morphological changes, and increased permeability. These results suggested that GE was similar to RhoA/ROCK inhibitor Y-27632, which inhibited the intervention of RhoA in FLSs via RhoA/p38MAPK/NF- $\mathrm{B} / \mathrm{F}$-actin signaling pathway and reduced the hyperpermeability of the FLSs, and thus playing a therapeutic role.

\section{CONCLUSION}

In summary, the mechanism of GE treatment of AA may be for two aspects. On the one hand, the expressions of 
pro-inflammatory cytokines IL-1 $\beta$ and IL-17 were downregulated and the expressions of anti-inflammatory cytokines IL4 andTGF- $\beta 1$ were up-regulated to restore the dynamic balance of pro-inflammatory cytokines and anti-inflammatory cytokines, thereby inhibiting the development of inflammation. On the other hand, GE inhibited LPS-induced RohA/p38MAPK/NF$\kappa \mathrm{B} / \mathrm{F}$-actin pathway in FLSs. GE attenuated LPS-induced stress fiber formation and increased permeability of FLSs via inhibition of the RohA/p38MAPK/NF- $\mathrm{B} / \mathrm{F}$-actin pathway. These observations indicated that GE may be an effective drug for treating RA in the clinic. Finally, our data suggested that the RohA/p38MAPK/NF- $\mathrm{B} / \mathrm{F}$-actin pathway may be a new target for the treatment of RA.

\section{REFERENCES}

Byng-Maddick, R., and Ehrenstein, M. R. (2015). The impact of biological therapy on regulatory $\mathrm{T}$ cells in rheumatoid arthritis. Rheumatology 54, 768-775. doi: 10.1093/rheumatology/keu487

Chakera, A., Bennett, S. C., Morteau, O., Bowness, P., Luqmani, R. A., and Cornall, R. J. (2012). The phenotype of circulating follicular-helper $\mathrm{T}$ cells in patients with rheumatoid arthritis defines CD200 as a potential therapeutic target. Clin. Dev. Immunol. 2012:948218. doi: 10.1155/2012/948218

Chang, Y., Sun, X., Jia, X., Xu, S., Wei, F., Yang, X., et al. (2015). Expression and effects of B-lymphocyte stimulator and its receptors in $\mathrm{T}$ cell-mediated autoimmune arthritis. Int. Immunopharmacol. 24, 451-457. doi: 10.1016/j. intimp.2015.01.001

Chen, C. X., Chen, J. Y., Kou, J. Q., Xu, Y. L., Wang, S. Z., Zhu, Q., et al. (2015). Suppression of inflammation and arthritis by orally administrated cardiotoxin from Naja naja atra. Evid. Based Complement. Alternat. Med. 2015:387094. doi: 10.1155/2015/387094

Chen, J., Wu, H., Xu, G. B., Dai, M. M., Hu, S. L., Sun, L. L., et al. (2015). Determination of geniposide in adjuvant arthritis rat plasma by ultra-high performance liquid chromatography tandem mass spectrometry method and its application to oral bioavailability and plasma protein binding ability studies. J. Pharm. Biomed. Anal. 108, 122-128. doi: 10.1016/j.jpba.2015. 01.044

Connolly, M., Veale, D. J., and Fearon, U. (2011). Acute serum amyloid A regulates cytoskeletal rearrangement, cell matrix interactions and promotes cell migration in rheumatoid arthritis. Ann. Rheum. Dis. 70, 1296-1303. doi: 10.1136/ard.2010.142240

Criado, G., Risco, A., Alsina-Beauchamp, D., Pérez-Lorenzo, M. J., Escós, A., and Cuenda, A. (2014). Alternative p38 MAPKs are essential for collagen-induced arthritis. Arthritis Rheumatol. 66, 1208-1217. doi: 10.1002/art.38327

Dai, M. M., Wu, H., Li, H., Chen, J., Chen, J. Y., Hu, S. L., et al. (2014). Effects and mechanisms of geniposide on rats with adjuvant arthritis. Int. Immunopharmacol. 20, 46-53. doi: 10.1016/j.intimp.2014.02.021

Dasgupta, S. K., Le, A., Vijayan, K. V., and Thiagarajan, P. (2017). Dasatinib inhibits actin fiber reorganization and promotes endothelial cell permeability through RhoA-ROCK pathway. Cancer Med. 6, 809-818. doi: 10.1002/cam4.1019

Dong, G. F., Zhang, X., He, D. N., Li, L., and Zhang, G. F. (2015). Effect of leflunomide on the abnormal expression of lipid rafts and F-Actin in B lymphocytes from patients with systemic lupus erythematosus. J. Immunol. Res. 2015:832916. doi: 10.1155/2015/832916

Dong, R., Hu, D., Yang, Y., Chen, Z., Fu, M., Wang, D. W., et al. (2017). EETs reduces LPS-induced hyperpermeability by targeting GRP78 mediated Src activation and subsequent Rho/ROCK signaling pathway. Oncotarget 8 , 50958-50971. doi: 10.18632/oncotarget.17331

Filer, A. (2013). The fibroblast as a therapeutic target in rheumatoid arthritis. Curr. Opin. Pharmacol. 13, 413-419. doi: 10.1016/j.coph.2013.02.006

Firestein, G. S. (2003). Evolving concepts of rheumatoid arthritis. Nature 423, 356-361. doi: 10.1038/nature01661

Firestein, G. S., and McInnes, I. B. (2017). Immunopathogenesis of rheumatoid arthritis. Immunity 46, 183-196. doi: 10.1016/j.immuni.2017.02.006

\section{AUTHOR CONTRIBUTIONS}

Participated in research design: RD, FL, and HW. Conducted the experiments: RD, FL, W-yW, LD, and Z-rZ. Contributed new reagents or analytic tools: $\mathrm{RD}, \mathrm{FL}$, and $\mathrm{LD}$. Performed the data analysis: RD, FL, and JF. Wrote or contributed to the writing of the manuscript: RD and FL.

\section{FUNDING}

This work was supported by grants from the National Natural Science Foundation of China (Nos. 81073122 and 81473400).

Fu, Y., Liu, B., Liu, J., Liu, Z., Liang, D., Li, F., et al. (2012). Geniposide, from Gardenia jasminoides Ellis, inhibits the inflammatory response in the primary mouse macrophages and mouse models. Int. Immunopharmacol. 14, 792-798. doi: 10.1016/j.intimp.2012.07.006

Ganesan, R., Doss, H. M., and Rasool, M. (2016). Majoon ushba, a polyherbal compound, suppresses pro-inflammatory mediators and RANKL expression via modulating NFKB and MAPKs signaling pathways in fibroblastlike synoviocytes from adjuvant-induced arthritic rats. Immunol. Res. 64, 1071-1086. doi: 10.1007/s12026-016-8794-x

Kawanami, D., Matoba, K., Kanazawa, Y., Ishizawa, S., Yokota, T., and Utsunomiya, K. (2011). Thrombin induces MCP-1 expression through Rhokinase and subsequent $\mathrm{p} 38 \mathrm{MAPK} / \mathrm{NF}-\kappa \mathrm{B}$ signaling pathway activation in vascular endothelial cells. Biochem. Biophys. Res. Commun. 411, 798-803. doi: 10.1016/j.bbrc.2011.07.031

Kim, E. K., Kwon, J. E., Lee, S. Y., Lee, E. J., Kim, D. S., Moon, S. J., et al. (2017). IL-17-mediated mitochondrial dysfunction impairs apoptosis in rheumatoid arthritis synovial fibroblasts through activation of autophagy. Cell Death Dis. 8:e2565. doi: $10.1038 /$ cddis.2016.490

Kong, Q. Z., Guo, L. T., Yang, J. N., Wang, Y. F., Zhao, J. X., Kong, S. H., et al. (2016). Anti-inflammatory effects of TRAF-interacting protein in rheumatoid arthritis fibroblast-like synoviocytes. Mediators Inflamm. 2016:3906108. doi: 10.1155/2016/3906108

Krabben, A., Wilson, A. G., de Rooy, D. P., Zhernakova, A., Brouwer, E., Lindqvist, E., et al. (2013). Genetic variants in the IL-4 and IL-4 receptor genes in association with the severity of joint damage in rheumatoid arthritis: a study in seven cohorts. Arthritis Rheum. 65, 3051-3057. doi: 10.1002/art.38141

Li, L., Li, G., Yu, C., and Li, Y. (2013). A meta-analysis of the role of p38 mitogenactivated protein kinase inhibitors in patients with active rheumatoid arthritis. Clin. Rheumatol. 32, 1697-1702. doi: 10.1007/s10067-013-2340-1

Liu, Y. R., Yan, X., Yu, H. X., Yao, Y., Wang, J. Q., Li, X. F., et al. (2017). NLRC5 promotes cell proliferation via regulating the NF- $\kappa \mathrm{B}$ signaling pathway in Rheumatoid arthritis. Mol. Immunol. 91, 24-34. doi: 10.1016/j.molimm.2017. 08.024

Lubberts, E. (2015). Role of T lymphocytes in the development of rheumatoid arthritis. Implications for treatment. Curr. Pharm. Des. 21, 142-146. doi: 10.2174/1381612820666140825122247

Lv, Q., Zhu, X. Y., Xia, Y. F., Dai, Y., and Wei, Z. F. (2015). Tetrandrine inhibits migration and invasion of rheumatoid arthritis fibroblast-like synoviocytes through down-regulating the expressions of Rac1, Cdc42, and RhoA GTPases and activation of the PI3K/Akt and JNK signaling pathways. Chin. J. Nat. Med. 13, 831-841. doi: 10.1016/S1875-5364(15)30087-X

Mankia, K., and Emery, P. (2015). Is localized autoimmunity the trigger for rheumatoid arthritis unravelling new targets for prevention. Discov. Med. 20, $129-135$.

Monickaraj, F., McGuire, P. G., Nitta, C. F., Ghosh, K., and Das, A. (2016). Cathepsin D: an $\mathrm{M} \phi$-derived factor mediating increased endothelial cell permeability with implications for alteration of the blood-retinal barrier in diabetic retinopathy. FASEB J. 30, 1670-1682. doi: 10.1096/fj.15-279802

Ng, C. T., Fong, L. Y., Sulaiman, M. R., Moklas, M. A., Yong, Y. K., Hakim, M. N., et al. (2015). Interferon-gamma increases endothelial permeability by causing 
activation of p38 MAP kinase and actin cytoskeleton alteration. J. Interferon Cytokine Res. 35, 513-522. doi: 10.1089/jir.2014.0188

Ni, Y., Teng, T., Li, R., Simonyi, A., Sun, G. Y., and Lee, J. C. (2017). TNF $\alpha$ alters occludin and cerebral endothelial permeability: role of p38MAPK. PLOS ONE 12:e0170346. doi: 10.1371/journal.pone.0170346

Pan, T., Shi, X., Chen, H., Chen, R., Wu, D., Lin, Z., et al. (2017). Geniposide suppresses interleukin-1 $\beta$-induced inflammation and apoptosis in rat chondrocytes via the PI3K/Akt/NF- $\mathrm{\kappa B}$ signaling pathway. Inflammation doi: 10.1007/s10753-017-0694-2 [Epub ahead of print].

Poli, A., Coleman, P. J., Mason, R. M., and Levick, J. R. (2002). Contribution of F-Actin to barrier properties of the blood-joint pathway. Microcirculation 9, 419-430. doi: 10.1038/sj.mn.7800149

Puthenedam, M., Williams, P. H., Lakshmi, B. S., and Balakrishnan, A. (2007). Modulation of tight junction barrier function by outer membrane proteins of enteropathogenic Escherichia coli: role of F-actin and junctional adhesion molecule-1. Cell Biol. Int. 31, 836-844. doi: 10.1016/j.cellbi.2007.01.036

Qian, C., Kuang, M., and Wang, Y. (2017). Effect of qianghuo erhuang decoction on $\mathrm{T}$ regulatory and $\mathrm{T}$ helper 17 cells in treatment of adjuvant-induced arthritis in rats. Sci. Rep. 7:17198. doi: 10.1038/s41598-017-17566-w

See, L. C., Kuo, C. F., Chou, I. J., Chiou, M. J., and Yu, K. H. (2013). Sexand age-specific incidence of autoimmune rheumatic diseases in the Chinese population: a Taiwan population-based study. Semin. Arthritis Rheum. 43, 381-386. doi: 10.1016/j.semarthrit.2013.06.001

Singer, I. I., Kazazis, D. M., Kawka, D. W., Rupp, E. A., and Bayne, E. K. (1985). Extracellular matrix-cytoskeletal interactions in rheumatoid arthritis. I. Immunoelectron microscopic analysis of the fibronexus at the adhesive surface of normal porcine type B synoviocytes in vitro. Arthritis Rheum. 28, 1105-1116. doi: 10.1002/art.1780281005

Stanley, A., Thompson, K., Hynes, A., Brakebusch, C., and Quondamatteo, F. (2014). NADPH oxidase complex-derived reactive oxygen species, the actin cytoskeleton, and Rho GTPases in cell migration. Antioxid. Redox Signal. 20, 2026-2042. doi: 10.1089/ars.2013.5713

Suzuki, T., Iwamoto, N., Yamasaki, S., Nishino, A., Nakashima, Y., Horai, Y., et al. (2015). Upregulation of thrombospondin 1 expression in synovial tissues and plasma of rheumatoid arthritis: role of transforming growth factor- $\beta 1$ toward fibroblast-like synovial cells. J. Rheumatol. 42, 943-947. doi: 10.3899/jrheum. 141292

Talaat, R. M., Mohamed, S. F., Bassyouni, I. H., and Raouf, A. A. (2015). Th1/Th2/Th17/Treg cytokinein systemic lupus erythematosus (SLE) patients: correlation with disease activity. Cytokine 72, 146-153. doi: 10.1016/j.cyto.2014. 12.027

Tan, Y., Qi, Q., Lu, C., Niu, X., Bai, Y., Jiang, C., et al. (2017). Cytokine imbalance as a common mechanism in both psoriasis and rheumatoid arthritis. Mdiators Inflamm. 2017:2405291. doi: 10.1155/2017/2405291

Walsh, D. A., McWilliams, D. F., Turley, M. J., Dixon, M. R., Fransès, R. E., Mapp, P. I., et al. (2010). Angiogenesis and nerve growth factor at the osteochondral junction in rheumatoid arthritis and osteoarthritis. Rheumatology 49, 1852-1861. doi: 10.1093/rheumatology/keq188

Wang, J., Liu, H., Chen, B., Li, Q., Huang, X., Wang, L., et al. (2012). RhoA/ROCKdependent moesin phosphorylation regulates AGE-induced endothelial cellular response. Cardiovasc. Diabetol. 11:7. doi: 10.1186/1475-2840-11-7

Wang, R., Wu, H., Chen, J., Li, S. P., Dai, L., Zhang, Z. R., et al. (2017). Antiinflammation effects and mechanisms study of geniposide on rats with collagen induced arthritis. Phytother. Res. 31, 631-637. doi: 10.1002/ptr.5775

Wang, Y., Wu, Q., Liu, Z., Guo, X., Zhou, L., Wang, Y., et al. (2017). A recombinant IgG-like bispecific antibody acting as interleukin-1 $\beta$ and interleukin-17A inhibitor exhibits a promising efficacy for rheumatoid arthritis. Biomed. Pharmacother. 89, 426-437. doi: 10.1016/j.biopha.2017.02.045

Wang, Z. C., Lu, H., Zhou, Q., Yu, S. M., Mao, Y. L., Zhang, H. J., et al. (2015). MiR-451 inhibits synovial fibroblasts proliferation and inflammatory cytokines secretion in rheumatoid arthritis through mediating p38MAPK signaling pathway. Int. J. Clin. Exp. Pathol. 8, 14562-14567.
Wei, F., Liu, S., Luo, L., Gu, N., Zeng, Y., Chen, X., et al. (2017). Antiinflammatory mechanism of ulinastatin: inhibiting the hyperpermeability of vascular endothelial cells induced by TNF- $\alpha$ via the RhoA/ROCK signal pathway. Int. Immunopharmacol. 46, 220-227. doi: 10.1016/j.intimp.2017. 03.007

Wei, W., Chen, M. Z., and Xu, S. Y. (1986). Pharmacological effects of isoxicam. Chin. Pharmacol. Bull. 2, 29-34.

Wu, H., Chen, Y., Wei, W., and Song, L.-H. (2008). Effects and mechanisms of treating adjuvant arthritis by total glucosides of cape jasmine in rat. Chin. J. Exp. Tradit. Med. Formul. 14, 49-52.

Xiao, Y., Liang, L., Pan, Y., Lian, F., Li, L., Lin, H., et al. (2013). Inhibitory effects of simvastatin on migration and invasion of rheumatoid fibroblast-like synoviocytes by preventing geranylgeranylation of RhoA. Rheumatol. Int. 33, 389-399. doi: 10.1007/s00296-012-2383-7

Xiaofeng, Y., Qinren, C., Jingping, H., Xiao, C., Miaomiao, W., Xiangru, F., et al. (2012). Geniposide, an iridoid glucoside derived from Gardenia jasminoides, protects against lipopolysaccharide-induced acute lung injury in mice. Planta Med. 78, 557-564. doi: 10.1055/s-0031-1298212

Xu, B., Li, Y. L., Xu, M., Yu, C. C., Lian, M. Q., Tang, Z. Y., et al. (2017). Geniposide ameliorates TNBS-induced experimental colitis in rats via reducing inflammatory cytokine release and restoring impaired intestinal barrier function. Acta Pharmacol. Sin. 38, 688-698. doi: 10.1038/aps.2016.168

Yin, Q., Xia, Y., and Wang, G. (2016). Sinomenine alleviates high glucose-induced renal glomerular endothelial hyperpermeability by inhibiting the activation of RhoA/ROCK signaling pathway. Biochem. Biophys. Res. Commun. 477, 881-886. doi: 10.1016/j.bbrc.2016.06.152

Yokota, S., Chosa, N., Kyakumoto, S., Kimura, H., Ibi, M., Kamo, M., et al. (2017). ROCK/actin/MRTF signaling promotes the fibrogenic phenotype of fibroblastlike synoviocytes derived from the temporomandibular joint. Int. J. Mol. Med. 39, 799-808. doi: 10.3892/ijmm.2017.2896

Yu, S. M., Cho, H., Kim, G. H., Chung, K. W., Seo, S. Y., and Kim, S. J. (2016). Berberine induces dedifferentiation by actin cytoskeleton reorganization via phosphoinositide 3-kinase/Akt and p38 kinase pathways in rabbit articular chondrocytes. Exp. Biol. Med. 241, 800-807. doi: 10.1177/1535370216631028

Zhang, C., Wu, Y., Xuan, Z., Zhang, S., Wang, X., Hao, Y., et al. (2014). p38MAPK, Rho/ROCK and PKC pathways are involved in influenza-induced cytoskeletal rearrangement and hyperpermeability in PMVEC via phosphorylating ERM. Virus Res. 192, 6-15. doi: 10.1016/j.virusres.2014.07.027

Zhang, Z. R., Wu, H., Wang, R., Li, S. P., Dai, L., and Wang, W. Y. (2017). Immune tolerance effect in mesenteric lymph node lymphocytes of geniposide on adjuvant arthritis rats. Phytother. Res. 31, 1249-1256. doi: 10.1002/ptr.5847

Zhuang, Y., Liu, J., Ma, P., Bai, J., Ding, Y., Yang, H., et al. (2017). Tamarixinin A alleviates joint destruction of rheumatoid arthritis by blockade of MAPK, and NF-kB activation. Front. Pharmacol. 8:538. doi: 10.3389/fphar.2017.00538

Zou, S., Wang, C., Cui, Z., Guo, P., Meng, Q., Shi, X., et al. (2016). $\beta$-Elemene induces apoptosis of human rheumatoid arthritis fibroblast-like synoviocytes via reactive oxygen species-dependent activation of p38 mitogen-activated protein kinase. Pharmacol. Rep. 68, 7-11. doi: 10.1016/j.pharep.2015.06.004

Conflict of Interest Statement: The authors declare that the research was conducted in the absence of any commercial or financial relationships that could be construed as a potential conflict of interest.

The reviewer YH and handling Editor declared their shared affiliation.

Copyright $\odot 2018$ Deng, Li, Wu, Wang, Dai, Zhang and Fu. This is an open-access article distributed under the terms of the Creative Commons Attribution License (CC BY). The use, distribution or reproduction in other forums is permitted, provided the original author(s) and the copyright owner are credited and that the original publication in this journal is cited, in accordance with accepted academic practice. No use, distribution or reproduction is permitted which does not comply with these terms. 